\title{
Properties of Cold-Pressed Metal Hydride Materials for Neutron Shielding in a D-T Fusion Reactor*)
}

\author{
Hiroaki MUTA, Teruya TANAKA ${ }^{1)}$, Yuji OHISHI, Ken KUROSAKI, Yoshimitsu HISHINUMA ${ }^{1)}$, \\ Shinsuke YAMANAKA and Takeo MUROGA ${ }^{1)}$ \\ Graduate School of Engineering, Osaka University, 2-1 Yamadaoka, Suita, Osaka 565-0871, Japan \\ 1) National Institute for Fusion Science, 322-6 Oroshi-cho, Toki, Gifu 509-5292, Japan
}

(Received 25 November 2014 / Accepted 2 February 2015)

\begin{abstract}
Thinner radiation shielding materials are desired for deuterium-tritium fusion reactors. Metal hydrides are considered one of the candidate materials because of their high hydrogen density. However, the bulk hydride is brittle and difficult to fabricate. In the present study, titanium- and zirconium-hydride pellets were prepared by cold pressing from their powders to simplify the fabrication process. The pressing strongly deforms the hydride particle; $\approx 90 \%$ of the theoretical density was achieved by cold pressing with the pressure of about $1 \mathrm{GPa}$. Thermal conductivities of the high-density pellets were comparable with those for the bulk metal hydrides. The results indicate that cold pressing is a promising way of fabricating dense metal hydrides.
\end{abstract}

(C) 2015 The Japan Society of Plasma Science and Nuclear Fusion Research

Keywords: titanium hydride, zirconium hydride, neutron shielding material, cold pressing, thermal conductivity

DOI: $10.1585 /$ pfr. 10.3405021

\section{Introduction}

In fusion reactors, thinner radiation-shield layers made of high-density neutron-shielding materials must be installed to reduce the size of reactors. Previous neutronics studies indicated that combinations of ferritic steel and boron carbide or ferritic steel and water, tungsten carbide, etc., can be candidates for the effective shielding materials $[1,2]$. Meanwhile, metal hydrides, such as titanium and zirconium hydrides, are also considered for the shielding material due to their high densities of hydrogen atoms. For example, the hydrogen amount per unit volume of a typical titanium hydride is about twice as large as that of liquid hydrogen. According to shielding calculations [3], several metal hydrides such as titanium, vanadium, and zirconium can be good neutron-shielding materials for the deuteriumtritium (D-T) fusion reactor. These hydrides show high shielding abilities, low radioactivity after irradiation, and they also have relatively low mass densities compared with the tungsten carbide.

However, fabrication of the bulk non-crack metal hydrides is difficult because the hydrides are generally very brittle. Usually, the hydrides are prepared by the absorption of hydrogen gas into the bulk metal. The hydrogenation causes a large volume change, which leads to cracking and fracturing. Figure 1 shows a $\mathrm{Zr}-\mathrm{H}$ binary-phase diagram with the normalized volume change. The volume change in the $\alpha$-zirconium to $\delta$-zirconium hydride phase transition reaches about $20 \%$, which causes the cracking and pulverization of the bulk metal and the hydride.

author'se-mail: muta@see.eng.osaka-u.ac.jp

*) This article is based on the presentation at the 24th International Toki Conference (ITC24).

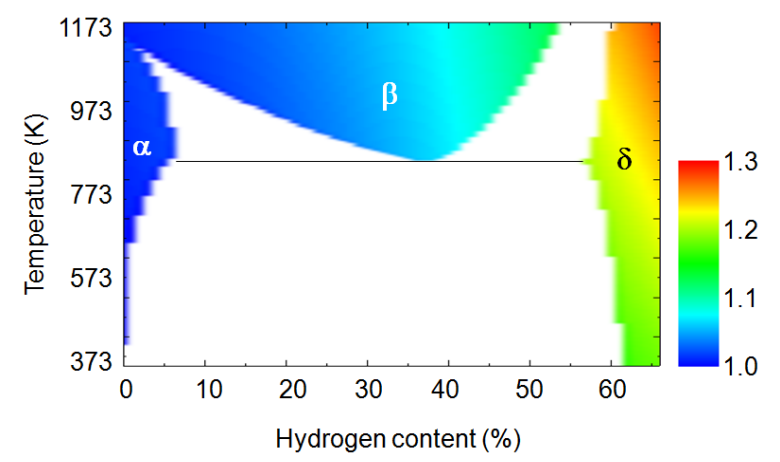

Fig. $1 \mathrm{Zr}-\mathrm{H}$ binary-phase diagram. $\alpha, \beta$, and $\delta$ are $\alpha$-zirconium, $\beta$-zirconium, and $\delta$-zirconium hydrides, respectively. The colors represent volume change from $\alpha$-zirconium.

Therefore, the temperature and time for the hydrogenation process should be carefully controlled with consideration of the volume change at the phase transition [4-10]. In the past, Yamanaka fabricated several centimeter-sized bulk hydrides, such as titanium and zirconium hydrides, $[4,5,7-10]$ using hydrogenation through $\beta$-phase zirconium to inhibit the volume change, which required several days. However, much larger sizes of hydrides are required for neutron-shielding materials in a D-T fusion reactor. The conventional process seems not to be suitable because of the long fabrication time and brittleness.

For the present study, we tried to make dense hydride pellets by cold pressing their powders to simplify the fabrication process. The hydride powders can be easily prepared and purchased commercially. If we can make dense 
hydride pellets by only the cold pressing, then the large neutron shield can be prepared by stacking the pellets. The shape change and pulverization may be neglected. From the aspect of physical properties, we measured the thermal conductivity of the hydride pellets. The thermal conductivity is an important parameter in the determination of the temperature distribution and is known to depend on the microstructure.

\section{Method}

Commercial $\varepsilon$-titanium hydride powder (Aldrich, 99\% purity) and $\varepsilon$-zirconium hydride powder (Aldrich, $99 \%$ purity) were used as starting materials. Figure 2 shows the scanning electron microscopy (SEM) images of the powder. The particle size is widely distributed from submicron to several tens of micrometers. The crystal structure was examined by X-ray diffraction (XRD) analysis (Ultima4, RIGAKU) before the cold pressing. The XRD patterns of the powders are shown in Fig. 3. Both XRD peaks correspond to those of the $\varepsilon$-phase of titanium or zirconium hydrides. No impurity phase was detected. The estimated lattice parameters from the XRD peaks were almost equal to those of $\mathrm{TiH}_{2.0}$ or $\mathrm{ZrH}_{2.0}$, hence the hydrogen/metal ratio was assumed to be 2.0 .

The powders were pelletized by cold pressing at pressures of 50-1220 MPa using tungsten carbide dies. The
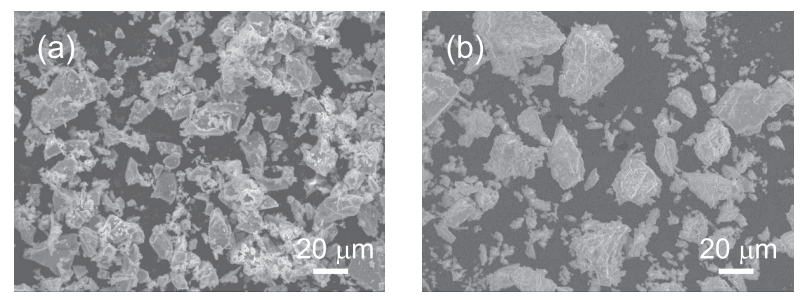

Fig. 2 SEM images of (a) titanium hydride and (b) zirconium hydride powders.

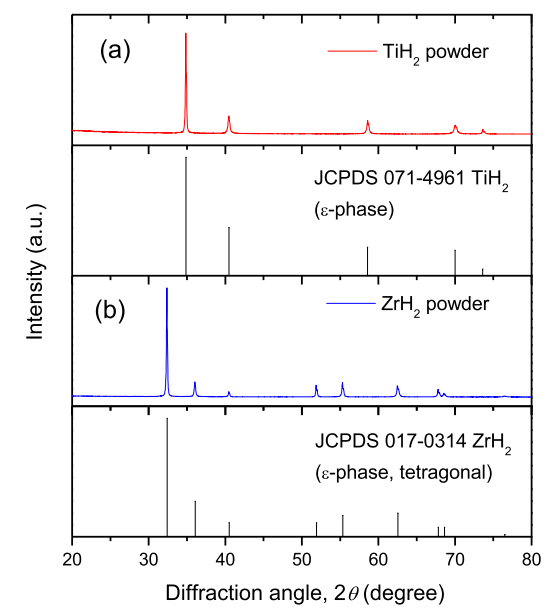

Fig. 3 XRD patterns of (a) titanium hydride and (b) zirconium hydride powders. pellets were of $10 \mathrm{~mm}$ diameter and about $2 \mathrm{~mm}$ thick. The density was calculated from the weight and dimensions. The pellet surface was observed by SEM (JSM-6500F, JEOL) and the attached electron backscatter diffraction (EBSD) analysis (OIM, TSL). The thermal diffusivity was measured by the laser flash method (LFA457, Netzsch) from room temperature to $623 \mathrm{~K}$. It was confirmed that hydrogen desorption did not occur in this temperature range. Using the literature data of the heat capacity $[8,9]$, the thermal conductivity, $\kappa$, was estimated by

$$
\kappa=\alpha C_{P} d,
$$

where $\alpha, C_{P}$, and $d$ are the thermal diffusivity, heat capacity, and sample density, respectively.

\section{Results and Discussion}

Figure 4 shows the pellet appearance of a cold-pressed zirconium hydride pellet. Similar to the zirconium hydride, the titanium hydride pellets are silver in color, not different from their corresponding metal forms. The dense pellets are not too brittle for the handling.

Figure 5 shows the SEM images of the pressed titanium hydride pellets. The pellet particle shapes remain the same as those of the starting powders with less than $200 \mathrm{MPa}$ pressure. The low-density pellets are easily broken by the bare hand. The pressing with more than $400 \mathrm{MPa}$ strongly deforms the particle shape and the pore

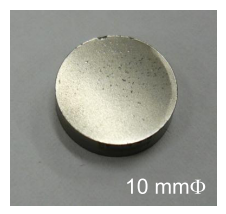

Fig. 4 A zirconium hydride pellet cold pressed using a pressure of $1220 \mathrm{MPa}$.
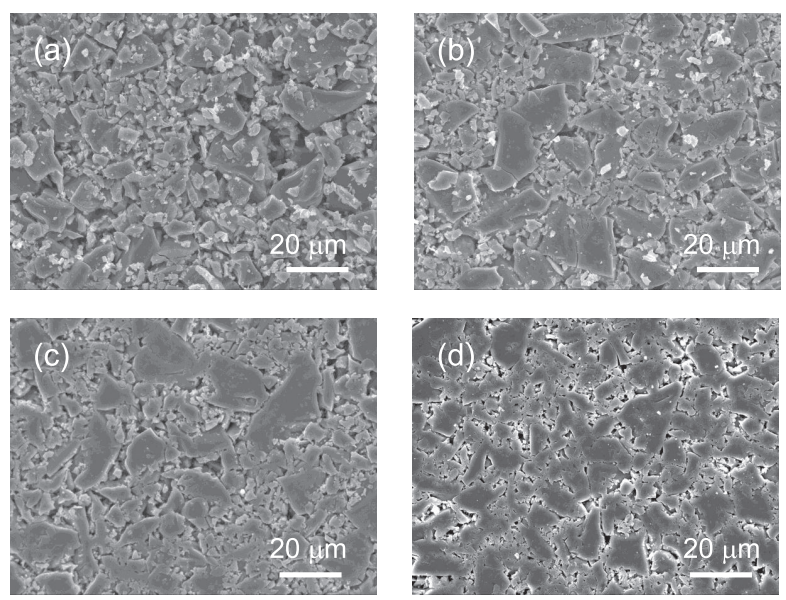

Fig. 5 SEM images of titanium hydride pellets pressed by pressure of (a) $50 \mathrm{MPa}$, (b) $200 \mathrm{MPa}$, (c) $400 \mathrm{MPa}$, and (d) $910 \mathrm{MPa}$. 

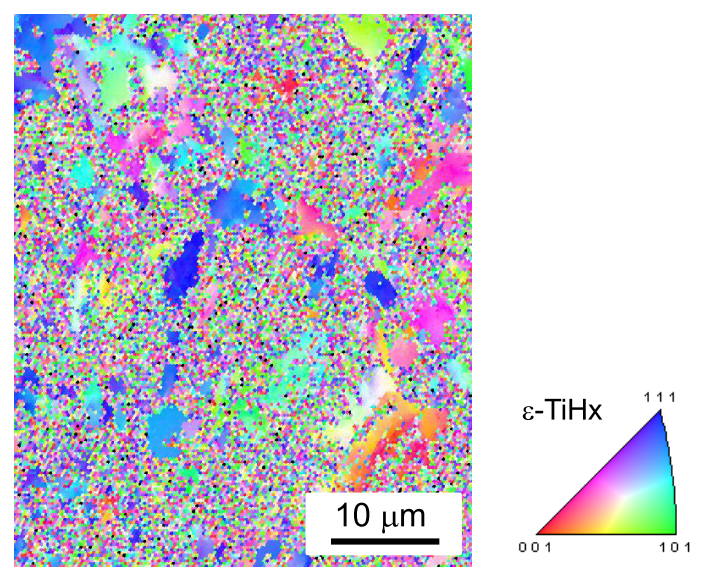

Fig. 6 IPF map of a titanium hydride pellet pressed by pressure of $400 \mathrm{MPa}$.

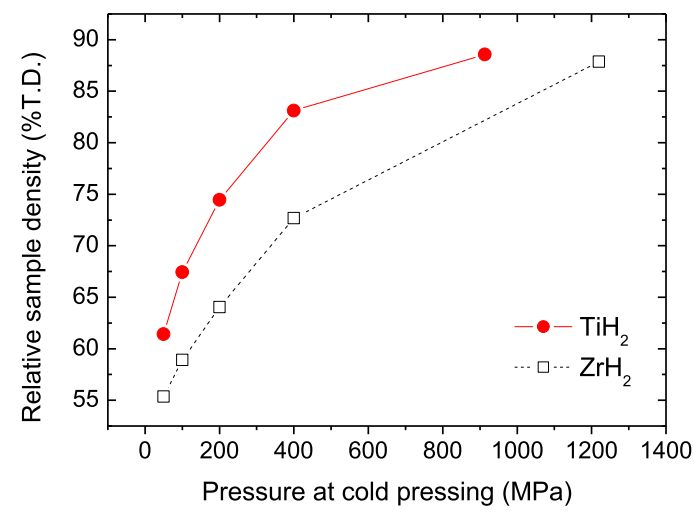

Fig. 7 Relation between pressure used in cold pressing and sample' s relative density.

region becomes small.

To estimate the particle deformation, EBSD observation was performed on the surface of the pellets. The inverse pole figure (IPF) map of a titanium hydride pellet pressed by a pressure of $400 \mathrm{MPa}$ is shown in Fig. 6. The color in the map reflects the crystal orientation of the particle. About a half region shows finely and randomly distributed colors due to the surface roughness and small particle diameter. The crystal orientation cannot be observed exactly in the area. Conversely, there are several small areas with similar colors. They show the hydride particle region. The color in each particle gradually changes. This means the crystal direction gradually changes, which indicates that these particles have elastic or inelastic strains $[11,12]$ caused by the cold pressing. Meanwhile, colors of the particle region seem to be randomly distributed, which indicate that the crystal texture is not formed for this sample.

Figure 7 shows the relation between the pressure used in the pressing and the sample's relative density for titanium and zirconium hydrides. The theoretical densities are $3.78 \mathrm{~g} \mathrm{~cm}^{-3}$ and $5.56 \mathrm{~g} \mathrm{~cm}^{-3}$ for $\varepsilon$-titanium and $\varepsilon$ -

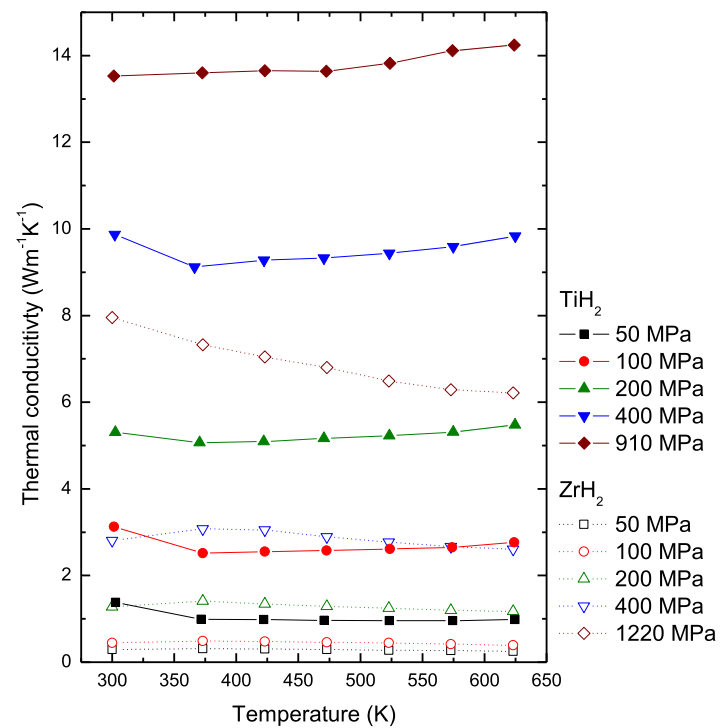

Fig. 8 Temperature dependence of thermal conductivity.

zirconium hydrides, respectively. With increasing pressures, the sample density increases as well. Titanium hydrides show higher relative densities compared to zirconium hydrides. The differences could be caused by differences in the raw-particle size and mechanical properties. About $90 \%$ of the theoretical density was achieved by cold pressing with about $1 \mathrm{GPa}$ pressure for both titanium and zirconium hydrides. This means that the thickness of a shield layer made of the present cold-pressed hydride will be thicker by $11 \%$ compared with that made of a hydride with one of the theoretical densities [2]. However, the density is considered acceptable because of the high shielding performance of hydrides. It is reported that the titanium is significantly softened by hydrogenation [5]. Young's modulus of $\mathrm{TiH}_{1.66}$ is about one third of that of titanium metal. The softening effect of hydrogenation might provide the high density only when cold pressed. Relatively large hydride powders were used in this study, so more densification can be expected by using fine powders.

The temperature dependence of thermal conductivity is shown in Fig. 8. The thermal conductivity increases with the pressure during the pressing. The thermal conductivity values of zirconium hydrides are less than those of titanium hydrides mainly because of the difference in the relative densities. Both hydrides with low relative sample densities show very small thermal conductivity. The temperature dependence is relatively weak, which indicates that the thermal conduction was dominated by the electrons.

Figure 9 shows the relation between the relative density and the thermal conductivity of the sample at room temperature. The thermal conductivity of bulk hydrides $\left(\mathrm{TiH}_{1.75}\right.$ and $\left.\mathrm{ZrH}_{1.65}\right)$ are plotted together. The thermal conductivity of the bulk sample is about $16-20 \mathrm{~W} \mathrm{~m}^{-1} \mathrm{~K}^{-1}$ $[9,10]$, which is almost the same as that of the conventional ferritic steel. For the pressed titanium hydride, which 


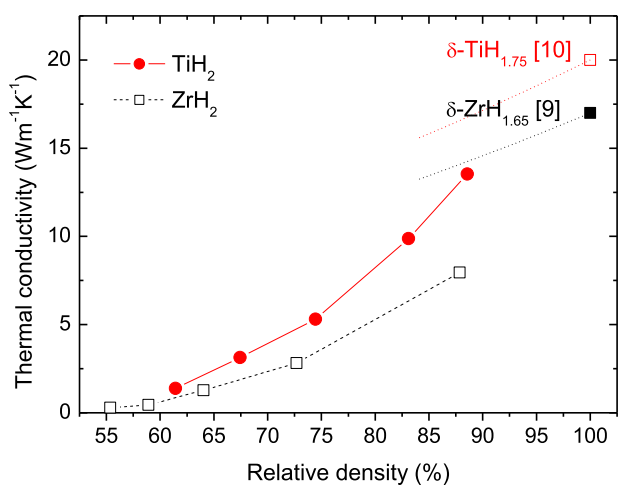

Fig. 9 Density dependence of the thermal conductivity. Values for $100 \%$ are literature data for each bulk metal hydride $[9,10]$.

has a relative density of $90 \%$, the thermal conductivity is $14 \mathrm{~W} \mathrm{~m}^{-1} \mathrm{~K}^{-1}$ which is comparable to that of the bulk hydride. The dotted line in the figure is the porosity dependence given by the Maxwell-Eucken equation [13];

$$
\kappa=\kappa_{0}(1-P) /(1+\beta P)
$$

where $\kappa_{0}, P$, and $\beta$ are the thermal conductivity without porosity, porosity, and the shape parameter, respectively. The parameter $\beta$ is assumed to be 0.5 , which corresponds to a spherical pore. The experimental thermal conductivities are lower than the expected values. The equation does not consider the grain boundary effect, so the difference might be attributed to the weak contact between the particles. Surface treatment would increase the thermal conductivity. However, the sample density and thermal conductivity for dense hydride samples are sufficiently high. A high sample density means a high density of hydrogen, that is, the neutron shielding materials. These results in- dicate that the hydride pellets fabricated by cold pressing can be a good candidate of the shielding material.

\section{Conclusion}

Titanium and zirconium hydride pellets were prepared by cold pressing from their powders. The density reached $\approx 90 \%$ of theoretical density for the pressing pressure of $\approx 1 \mathrm{GPa}$. The particle strongly deformed by the pressing.

The thermal conductivity was evaluated by the laser flash method. The value increased with increasing sample densities, reaching $14 \mathrm{~W} \mathrm{~m}^{-1} \mathrm{~K}^{-1}$ for the titanium hydride. The value is comparable with those of the bulk materials. The sufficient values of the sample density and thermal conductivity indicate that cold pressing is a promising way of fabricating metal hydrides, which are candidate materials for neutron shielding.

[1] T. Tanaka et al., J. Nucl. Sci. Technol. Supplement 6, 120 (2008).

[2] Y. Chen, FZKA 6763, Forschungszentrum Karlsruhe GmbH, Karlsruhe, 2003.

[3] T. Tanaka et al., presented at 21st Topical Meeting on the Technology of Fusion Energy (TOFE), Nov. 9-13, 2014, Anaheim, CA, USA. To be submitted to Fusion Science and Technology.

[4] S. Yamanaka et al., J. Alloys Compd. 293-295, 23 (1999).

[5] S. Yamanaka et al., J. Alloys Compd. 330-332, 99 (2002).

[6] B. Tsuchiya et al., J. Alloys Compd. 330-332, 357 (2002).

[7] D. Setoyama et al., J. Alloys Compd. 381, 215 (2004).

[8] D. Setoyama et al., J. Nucl. Mater. 344, 298 (2005).

[9] M. Uno et al., J. Alloys Compd. 366, 101 (2004).

[10] M. Ito et al., J. Alloys Compd. 420, 25 (2006).

[11] A.J. Wilkinson and D.J. Dingley, Acta Metal. Mater. 39, 3047 (1991).

[12] M. Kamaya et al., Nucl. Eng. Des. 235, 713 (2005).

[13] W.D. Kingery, H.K. Howen, D.R. Uhlmann (Eds.), Introduction to Ceramics, 2nd ed. (John Wiley \& Sons, New York, 1976). 